Some of the cultural methods to which we gave attention last year were the plowing under of a heavy crop of green rye before transplanting time; a comparison of close, medium and wide setting in the drill, and deep as compared with shallow cultivation. The indications were that for a dry season it is best to have the rye plowed under early, before it gets too large a growth. If the season remains good throughout the growing period, the rye will not be harmful even though it gets considerable growth before it is turned under.

For the average season and soil, about 18 inches in the drill and three and one-half feet apart for the rows gives the best results.

In connection with the breeding plat in Christian County, Ky., a fertilizer experiment was made which is a continuation of the work done in this section in former years. The 1908 results agree with hose obtained the previous year. The tenth acre plats receiving phosphoric acid either alone or in combination with potash and nitrogen, or both, gave good results, while the plats which received potash, nitrogen, or both, without phosphoric acid, were but little better than the check plats. We had previously found by chemical analysis that this soil was deficient in phosphoric acid. These results are mentioned to illustrate the importance of the plant breeder knowing the needs of the soil, and the chief elements that enter into the growth of the plant in order that he may get the highest development of the plant under all conditions whether favorable or otherwise.

\title{
THE PRODUCTION OF A NEW STRAIN OF TOBACCO AND ITS DEVELOPMENT.
}

Br J. B. STEwart, Tariffiville, Conn.

The word strain in plant breeding is used to designate a plant and its progeny, which has some-special feature that distinguishes it from the variety to which it belongs.

The feature which sets a plant apart and makes it a strain may pertain to either the foliage, flower or fruit, but not to all of them; for then the plant would be a new variety instead of a strain.

The word strain is also applied to a certain feature in a variety that makes it especially adapted to local conditions.

A new strain of any variety may be produced either by selection or hybridization, In most varieties of plants strains are easily produced, In fact, they are continually producing themselves by natural selection, which adapts the variety to local sojl and climate conditions. 
Production of a new strain by place effect. The production of a new strain in the varieties of the species Nicotiana Tabacum is not a difficult problem. By a change of soil or climate conditions the variety is easily influenced to break up into many strains. Especially is this true when the seeding of the variety is transported from a semi-tropical to a temperate climate. The fact has been demonstrated to the writer on several different occasions, when seed of the cigar leaf wrapper variety of tobacco has been transported from the island of Cuba to the Connecticut Valley. In each instance I have been able to select in a field of tobacco grown in the Connecticut Valley from seed imported from Cuba several strains of tobacco, some of which have proved of great value commercially when developed.

In the selection of a strain of tobacco for commercial purposes in a field where there is a general breaking up in the variety from place effect there are several major and minor points which must be taken into consideration. The major points consist of the characteristics which are more or less fixed in the strain from the beginning and can not be changed by subsequent development; while the minor ones are easily influenced by breeding and culture. The major and minor points will vary with the object for which the new strain is to be produced.

In selecting a new strain of tobacco to be grown in the Connecticut Valley, under shade, for wrapper purposes, the following points would be considered major characteristics:

1. Habit of growth of the plant, that is the general style of the plant.

2. The position of the axis of the leaf to the stalk, whether drooping or upright.

3. The relative variation in the size of the bottom and top leaves with respect to the middle ones.

4. The number and general shape of the leaves on the stalk.

5. The tendency of the plant to produce suckers or branches at the axes of the leaves.

The points which might be considered minor characteristics are:

1. The size of the leaves.

-2. Color and venation of leaves.

3. Tendency to rust or disease.

4. Quality of the leaf as to grain and burn.

When a plant which is to be a new strain of tobacco is selected according to the above field characteristics the seed is carefully saved free from cross pollination and the leaves are harvested when ripe. The leaves and the seed are labeled with corresponding numbers or labels, 
so that after the leaves, which we shall call the fruit of the tobacco plant, have been cured and fermented, the seed which belong to any certain bunch of leaves can be known and used to propagate and develop any special feature that may be desirable for a strain.

The Production of a new strain by hybridization. The tobacco flower is naturally self-fertilizing. The seed head is so arranged on the plant

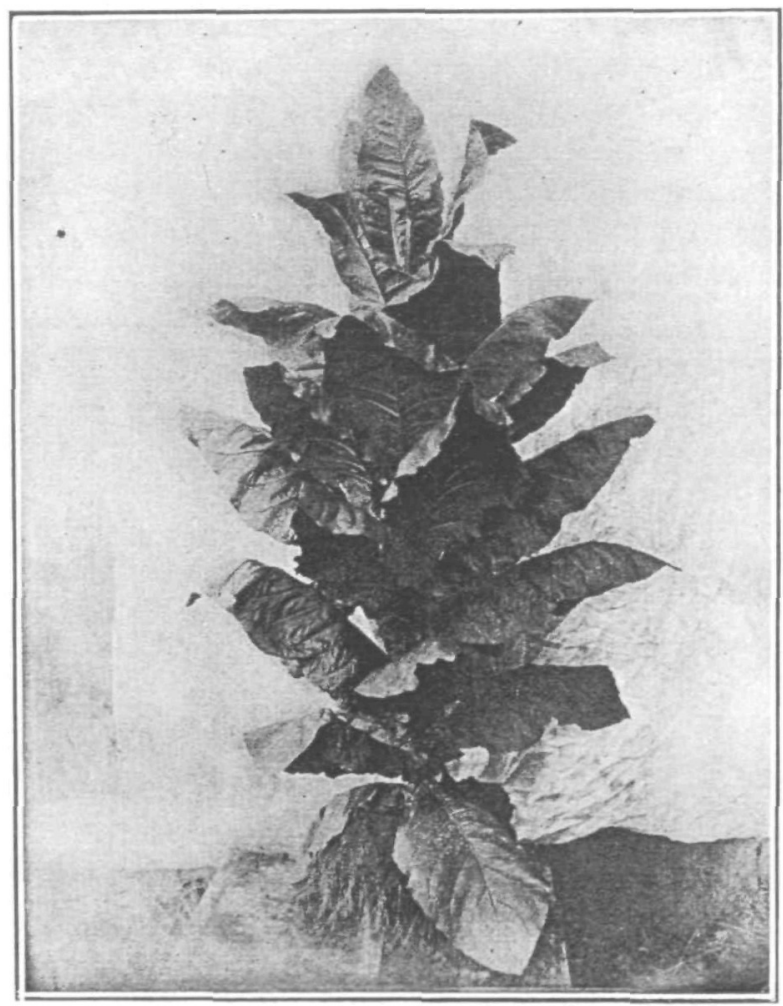

HaLladay haVANA SEED PLANT.

that it can be easily covered and protected from any possibility of cross pollination. The flower is large and so arranged that it is easy to dissect, emasculate and pollinate. When thoroughly pollinated a singleseed pod will produce nearly 500,000 seed. For these reasons the cross pollination of varieties of tobcaco is a very simple process, and the beginning of the production of a new strain of tobacco by hybridization is mere child's play, so far as the mechanical operation is concerned. 
The production of a new strain of tobacco of commercial value from such a cross between two varieties of tobacco is, however, more or less a game of chance, because it is impossible to grow all of the seed produced by the cross and each is capable in the third or fourth generation of producing a distinct strain of tobacco, a very few of which are of commercial importance. If, for instance, 200 plants are grown from every seed pod pollinated and the third generation is reached before the new

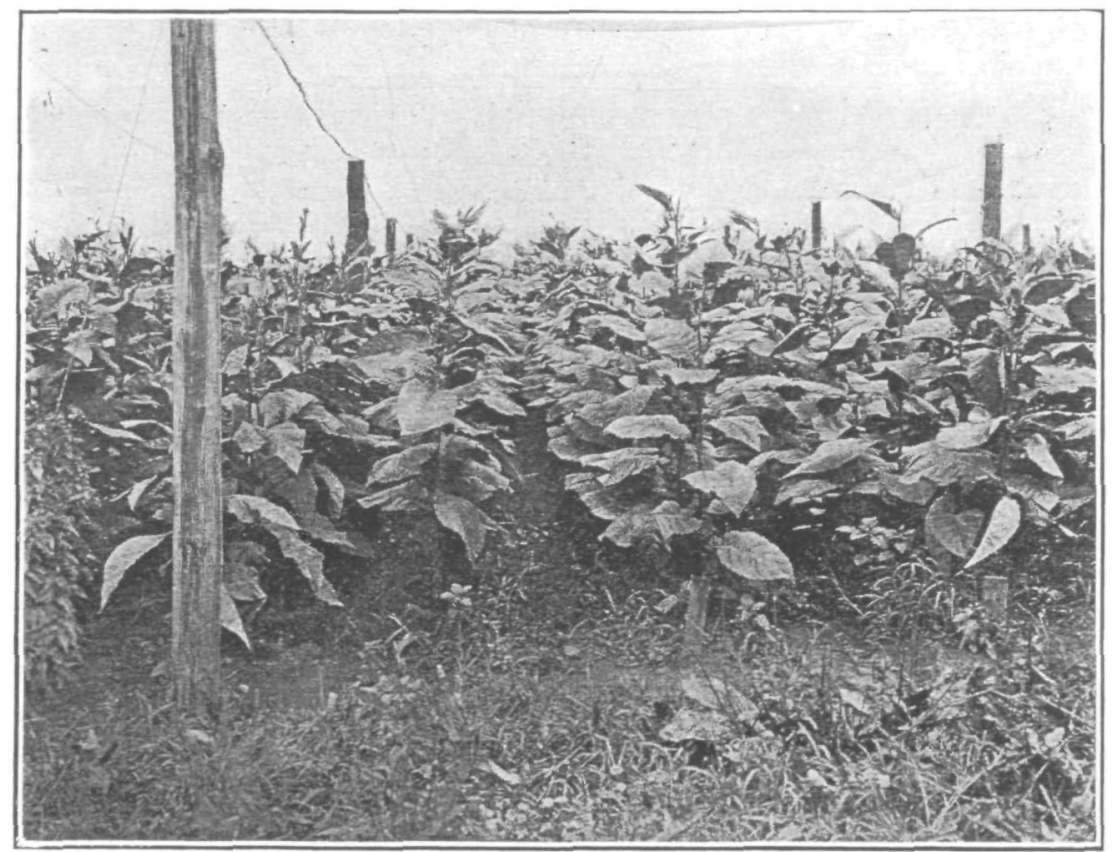

FIELD OF CUBAN TOBACCO, SHOWING IMPROVEMENT BY SELECTION.

strains appear, the chance of getting just the right plant is 1 to $1,000,000$. The fruit from these hybrids is iwild and of comparatively no value. For this əxason the production of a new strain of tobacco by hybridization is both costly and tedious.

From many thousands of hybrids between different varieties of tobacco the writer has only succeeded in producing one strain that promises to be of any extraordinary commercial value and it is an account of the production of this new strain of tobacco which has been 
named Halladay Havana, in honor of the farmer who has done much in the way of its development, that I wish to relate here:

The Halladay Havana is the result of the pollination of the Common Connecticut Havana tobacco with pollen from a strain of Sumatra tobacco, known as the smooth leaf type.

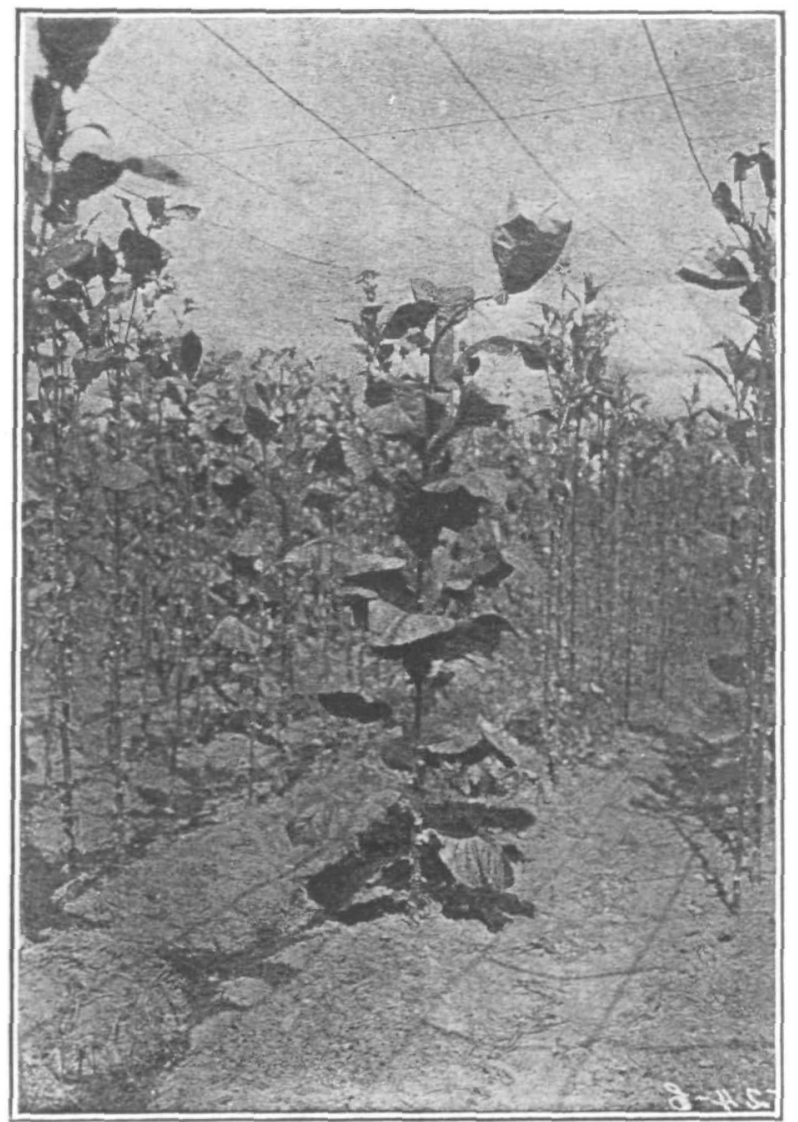

gumatra plant. Mate parent of the halladiy havaNa tobacco.

The Connecticut Havana is a variety of tobacco extensively grown in the Connecticut Valley for cigar wrapper purposes. It is a coarse, large, heavy leaved tobacco and with the introduction of the finest grades of tobacco, such as Sumatra, Cuban and Shade Grown, it was doomed to the binder or cheaper grade class unless it could be improved 
some way, so that the wrapper would be finer and of a higher grade. Fig. No. 1 gives an illustration of the Connecticut Havana seed. This variety of tobacco was crossed with the small, smooth leaf variety of the Sumatra type to produce a medium sized fine leaf.

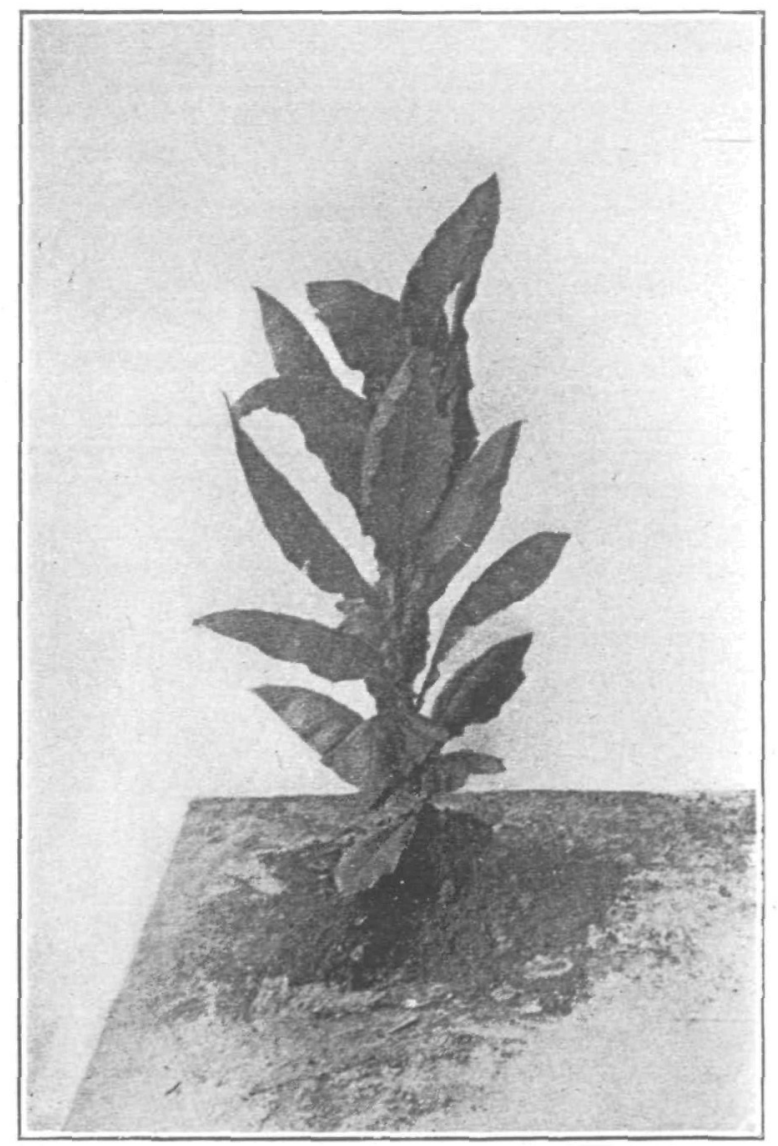

CoOley havana Seed, female PaRent of halladay havana seed.

The first and second generations of this hybrid produced a very large vigorous growing plant, with coarse, worthless fruit. In the third generation there was a general breaking up of this thrifty type and several distinct strains were isolated. Some of which remained true to type among these new strains was the Halladay Havana. 
The Halladay Havana is a medium sized leaf, erect habit of growth, with leaves set very close on the stalk, so that where the Common Connecticut Havana bears only from 16 to 20 leaves to the stalk, it bears from 26 to 30 . For this reason the yield was increased from the start 50 per cent over the ordinary variety. The quality of the fruit produced from this strain was at first coarse, variegated in color and very heavy in texture.

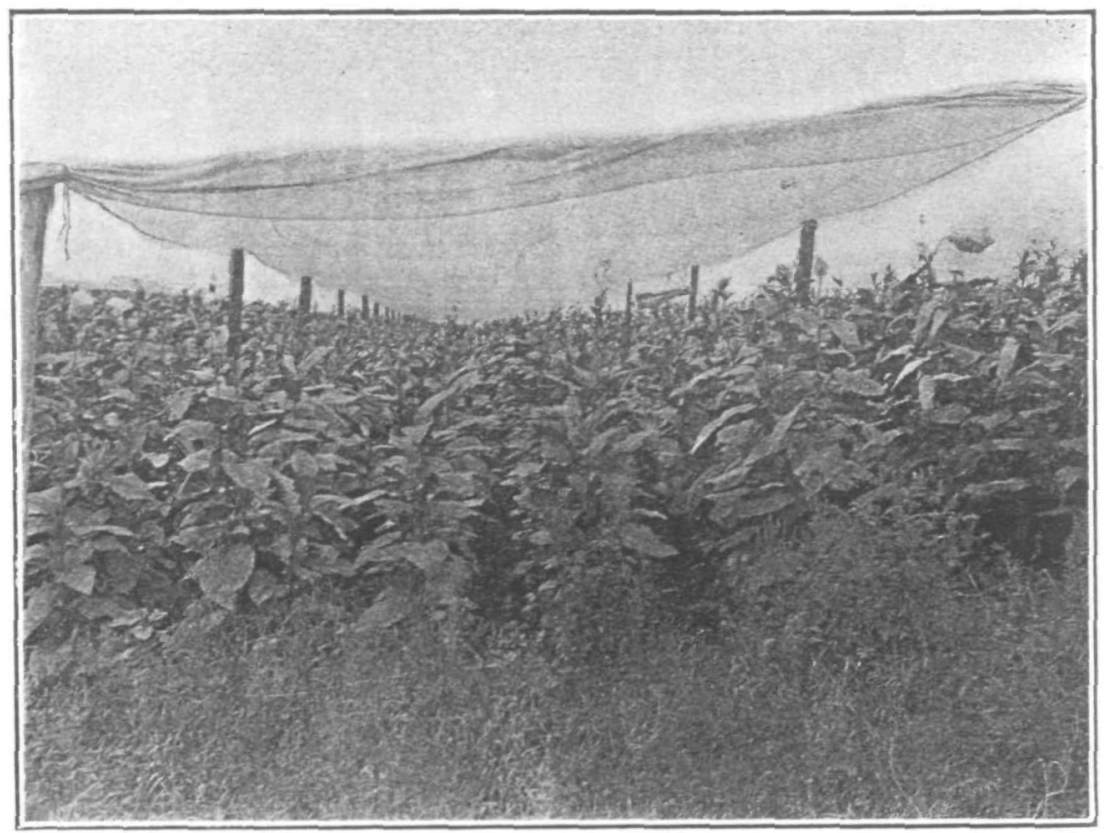

FIELD OF UNIMPROVED CUBAN TOBACCO. SHOWS THE BREAKING UP FROM PLACE EFFECT.

Developing a new strain of tobacco. The development of a new strain of tobacco is a slow and tedious process. It is here that the breeder must be an expert of sound judgment on the cured product. Unlike other plants the weight of the fruit of tobacco is not always a criterion that the new strain is always a commercial success. The leaves have to be put through a process of curing and fermentation that is extremely delicate and has much influence upon the commercial value of the product. This process is fully explained in Bulletin No. 138, Bureau of Plant Industry, U. S. Dept. of Agriculture. 
The method used in developing a new strain of tobacco is the plat test. The plants produced from a selected strain are set in a plot in the field and selections are made from these. The seed and fruit from the plants selected are labeled so that they can be associated with each other at any future period for further development. These plants on the plat which are not selected for seed are harvested and their fruit used as a commercial test of the new strain. The next season a plat is grown from each plant of the previous year's selections that promises any special merit. In this way the new strain is developed until it has either been discarded or become of commercial value.

- The second generation of the Halladay Havana showed some improvement in quality and the third generation, which was produced in 1908, is of fair quality; while the yield remains as high as 3310 pounds per acre. It is evident from the progress made in the improvement of this selection that within a very few years it will be the leading strain of tobacco_grown in the Connecticut Valley.

The development of the Hazlewood Cuban strain of tobacco for production under shade has been completed and can be shown in the following table, taken from Bulletin Nn. 138, Bureau of Plant Industry, U. S. Department of Agriculture;

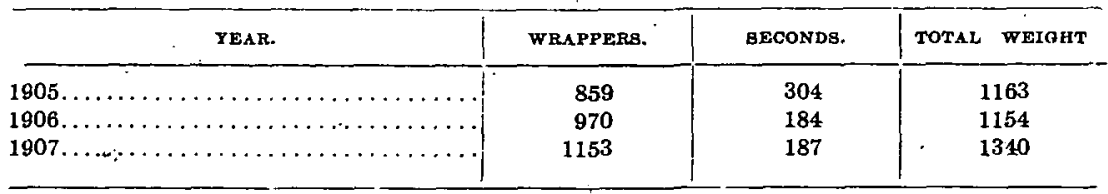

The development of this strain of tobacco has made profitable the production of a very high grade of wrapper tobacco under shade in the Connecticut Valley.

Conclusion. In conclusion I have only to say that the production and development of new strains of tobacco offers a fine field in which to study the hereditary tendency in plants, and as above stated, a new strain of tobacco has been produced that makes it possible for the Connecticut farmer to produce one and one-half tons of tobacco where heretofore he has had difficulty in producing 1 ton of commercial tobacco.

The development of a new strain of tobacco requires time, perseverance and a thorough knowlege of the plant and its cured product. 